\title{
Internet y redes sociales: un desafío a la convivencia familiar
}

\section{The Internet and social networks: a challenge to family coexistence}

\author{
Isabel Dans Álvarez De Sotomayor ${ }^{1}$ \\ isabel.dans@usc.es \\ Pablo César Muñoz Carril \\ pablocesar.munoz@usc.es \\ Universidade de Santiago de Compostela, España
}

\section{Resumen:}

Este artículo explora cómo internet y las redes sociales pueden convertirse en el origen de algunos conflictos en la esfera familiar. Se analiza la influencia de Internet en la convivencia familiar, examinando las preocupaciones y necesidades para educar en el ámbito digital. Se ha desarrollado un estudio cuantitativo no experimental empleando el método de encuesta. Participaron 1144 estudiantes de educación secundaria de la ciudad de A Coruña, todos ellos usuarios de internet y redes sociales. Un $76 \%$ de los encuestados manifiesta que sus padres no les prohíben nada cuando navegan por la red. Entre las principales acciones que los progenitores impiden realizar a sus hijos e hijas, se encuentran: realizar compras en línea, dar información personal y enviar correos a personas desconocidas. También se ha podido identificar que un $49 \%$ de los menores discuten con sus padres por el uso de internet. Asimismo, se constata que las mujeres, frente a los hombres, son las que afirman que su familia se preocupa en mayor medida por el tipo de contenidos a los que acceden, así como por los contactos incluidos en sus redes sociales. Es necesa-

\begin{abstract}
:
This article explores how the Internet and social networks can become the source of conflicts in the family sphere. The influence of the Internet on family life is analyzed, examining the concerns and needs when it comes to education in the digital world. A non-experimental quantitative study was carried out using the survey method. 1144 high school students from the city of A Coruña participated, all of them Internet and social media users. $76 \%$ of those surveyed state that their parents do not forbid them anything when they surf on the Net. The main actions that parents prevent their sons and daughters from doing include making online purchases, giving personal information and sending emails to strangers. It was also identified that $49 \%$ of minors argue with their parents about using the Internet. Likewise, it was found that women, in contrast to men, affirm that their family cares more about the type of content they access, as well as the contacts included on their social networks. It is necessary for parents to become aware of the importance of educating their children in a responsible use of technology. In this sense, acquiring adequate digital edu-
\end{abstract}

1 Dirección para correspondencia (correspondence address):

Isabel Dans Álvarez De Sotomayor. Departamento de Pedagogía y Didáctica. Universidade de Santiago de Compostela. Facultade de Ciencias da Educación. Rúa Prof. Vicente Fráiz Andón, s/n. Campus Vida. 15782 - Santiago de Compostela (España). 
rio que padres y madres sean conscientes de la importancia que supone educar a sus hijos/as en un uso responsable de la tecnología. En este sentido, adquirir una adecuada educación digital en el seno familiar resulta clave para minimizar conflictos, así como prevenir y alertar de los riesgos derivados de un uso inadecuado de internet y de las redes sociales.

\section{Palabras clave:}

Internet; redes sociales; familia; estudiantes de secundaria. cation within the family is key to minimizing conflicts, as well as preventing and becoming aware of the risks derived from an inappropriate use of the Internet and social networks.

\section{Key words:}

Internet; social networks; family; students of secondary education.

\section{Résumé}

Cet article explore comment l'internet et les réseaux sociaux peuvent devenir l'origine de certains conflits dans la sphère familiale. L'influence $d^{\prime}$ Internet sur la coexistence familiale est analysée, en examinant les préoccupations et les besoins d'éducation dans la sphère numérique. Une étude quantitative non expérimentale a été développée en utilisant la méthode de l'enquête. Un total de 1144 élèves de l'enseignement secondaire de la ville de La Corogne, tous utilisateurs d'Internet et de réseaux sociaux, ont participé à l'étude. $76 \%$ des personnes interrogées ont déclaré que leurs parents ne leur interdisent rien lorsqu'ils surfent sur le net. Parmi les principales actions que les parents empêchent leurs enfants de faire figurent: les achats en ligne, la communication d'informations personneIles et l'envoi d'e-mails à des personnes inconnues. II a également été identifié que 49\% des enfants se disputent avec leurs parents à cause de leur utilisation de l'internet. De même, il a été constaté que les femmes, par rapport aux hommes, sont celles qui déclarent que leur famille se préoccupe davantage du type de contenu auquel elles accèdent, ainsi que des contacts inclus dans leurs réseaux sociaux. Il est nécessaire que les parents soient conscients de l'importance d'éduquer leurs enfants à une utilisation responsable de la technologie. En ce sens, l'acquisition d'une éducation numérique adéquate au sein de la famille est essentielle pour minimiser les conflits, ainsi que pour prévenir et alerter des risques dérivés d'une utilisation inappropriée d'Internet et des réseaux sociaux.

\section{Mots clés:}

Internet; réseaux sociaux; famille; élèves du secondaire.

Fecha de recepción: 13-01-2021

Fecha de aceptación: 03-03-2021

\section{Introducción}

La era digital provoca grandes desafíos en la educación de los adolescentes. Las relaciones personales de jóvenes están mediadas, mayoritariamente, por la tecnología y tienen lugar, en gran medida, en nuevos ámbitos de interacción como las redes sociales. Además, frente al uso instrumental preferente de la tecnología por parte de los adultos, destaca un empleo lúdico y socializador en la juventud (Garrido, Busquet 
y Munté-Ramos, 2016). En este sentido, las redes sociales constituyen verdaderos contextos de desarrollo social, donde los jóvenes configuran su identidad, reciben las influencias de los modelos culturales y nutren su personalidad, sobre todo cuando son empleadas como fuentes prioritarias de información y conocimiento. Así, las redes sociales llegan a concentrar los múltiples escenarios de la formación presencial en un solo espacio y son concebidas, simultáneamente, como lugares de diversión, aprendizaje e intercambio social. Siguiendo con la metáfora de un único espacio que reúne varios escenarios, se puede aludir también a las redes sociales como una enorme sala de espejos, donde los adolescentes se encuentran con fragmentos de la sociedad y, al mismo tiempo, se reflejan a sí mismos.

Esta fragmentación de espacios queda reflejada en la diversidad de estudios que se están realizando sobre educación y juventud referidos a las tecnologías y, que en un esfuerzo de síntesis, podrían articularse considerando tres áreas fundamentalmente: aportes y limitaciones de la tecnología en los procesos de enseñanza-aprendizaje (Manca y Manieiri, 2015; Selwyn, 2016; Aaron y Lipton, 2018; Gallardo, De Castro y Saiz, 2020), peligros en la salud y en la seguridad (Terán, 2019; LozanoBlasco y Cortés-Pascual, 2020; Browne et al., 2019) y análisis de los procesos de identidad que se propician a través del uso intensivo de las aplicaciones tecnológicas (Min, 2016; Bernal y Konig, 2017). En estos y otros estudios, las conclusiones apuntan a la necesidad de una alfabetización digital como garantía de un uso apropiado de internet y las redes sociales en la adolescencia.

Efectivamente, la brújula para moverse por los entornos virtuales suele agruparse bajo la denominación de "alfabetización digital". La UNESCO (2011, p. 185) la define como "la habilidad de utilizar tecnología digital, herramientas de comunicación o redes para localizar, evaluar, usar y crear información". Pueden delimitarse diversas dimensiones a la hora de promover su alfabetización, sobre todo, teniendo en cuenta la educabilidad de las acciones digitales. La alfabetización mediática y digital (Hobbs, 2010, p.17), también conocida como AMI, abarca toda la gama de competencias cognitivas, emocionales y sociales que incluye el uso de textos, herramientas y tecnologías; las habilidades de pensamiento y el análisis crítico; la práctica de la composición y de la creatividad del mensaje; la capacidad de relacionarse con la reflexión y el pensamiento ético; así como la participación activa a través del trabajo en equipo y 
la colaboración. Algunos autores (Bartsch y Dienlin, 2016) destacan la alfabetización de la privacidad, que sería más necesaria para quienes permanecen en la red más tiempo y precisan de mayor protección ante las amenazas y la sensación de falsa seguridad que pueden sentir.

A quienes intervienen en este proceso de alfabetización se les conoce como mediadores, junto con la propia herramienta o medio social de comunicación. Colaboran con los adolescentes en el rol de "mediadores" otros jóvenes iguales en edad y estilo de vida; sus docentes, cuya acción suele ser escasa, aunque cada vez más demandada (Harper, 2018); y, sobre todo, sus familias (Dans, Muñoz y González, 2019). Las creencias de los colegiales sobre su propia educación digital, y las áreas de conflicto que suscitan, forman parte de un proceso formativo que va orientado a posibilitar su empoderamiento, dándoles voz y aportando luz sobre cómo planificar la mediación familiar en las redes sociales.

\section{Marco teórico}

\section{Internet como fuente de conflictos}

Los espacios virtuales están diseñados de un modo atractivo y seductor, de tal manera que logran retener mucho tiempo conectado a quien accede. Puede afirmarse que el diseño de la herramienta busca un efecto llamada y su propósito es mantener al usuario conectado el mayor tiempo posible. La creación de un estado afectivo positivo retiene al usuario en la navegación (Mauri, Cipresso, Balgera, Villamira, Riva, 2011; SerranoPuche, 2016). Esta cualidad afecta directamente al tiempo de conexión, que constituye una de las mayores objeciones de las familias ante el uso de Internet por sus hijos (Shin y Kang, 2016; Smahel et al., 2020). Los padres dan más valor a la presencialidad en las relaciones familiares, aunque reconocen que también sufren y/o disfrutan de la seducción de las redes sociales. La sobreexposición a las pantallas se considera perjudicial y es por ello que divide a las familias, aunque el origen de esta permanencia digital está presente, y resulta consustancial, en el propio diseño de las herramientas digitales. Junto a ello conviene considerar que este tiempo sobreabundante es criticado porque se entiende la red como una pérdida de tiempo o una fuente de distracción. Aunque, tal y como indican algunos autores, tanto las distracciones que suscita el dis- 
positivo como las preocupaciones sobre el tiempo dedicado a las pantallas, se detectan entre padres y adolescentes por igual (Pew Research Center, 2018; Michavila, Abad y García, 2019).

A esta concepción de los espacios digitales subyace una visión lúdica de las redes, que suele entenderse separada y, en ocasiones limitadora, del aprendizaje. Si bien existen factores que impulsan su aprovechamiento educativo (González-Sanmamed, Muñoz y Dans, 2017), es evidente que "un uso generalizado y positivo de las TIC en el proceso de enseñanza-aprendizaje supone inevitablemente la continua interacción entre los agentes involucrados: docentes, estudiantes y familias" (Gallardo, De Castro y Saiz, 2020, p. 133). Esta generalización constituye un hecho de gran actualidad por la situación mundial forzosa de escolarización a distancia debido a la pandemia generada por el COVID-19.

Si bien es cierto que el uso de Internet por los menores suele percibirse como conflictivo por el empleo de tiempo que suele acaparar, hay que tener en cuenta además una larga lista de otros problemas, y diversos riesgos, a los que se enfrentan los adultos y sus hijos en relación con las aplicaciones digitales. Estos inconvenientes han sido recogidos en diversas investigaciones (Vanderhoven, Schellens y Valcke, 2014; Christofides, Muise y Desmarais, 2012; Smahel et al., 2020) y existen guías al alcance de los educadores para afrontarlos. Una de las entidades públicas que proporciona formación sobre mediación parental es la Agencia Española de Protección de Datos (2016), quien señala estas áreas prioritarias:

a) "Contenidos inapropiados. Imágenes o información que les resulta dañina por su edad, madurez, sensibilidad, o por la propia temática o su tratamiento.

b) Pérdida de privacidad. Publicación excesiva de información privada que podría ser utilizada en su contra.

c) Incorrecta gestión de información de terceros. Problemas por publicar o reenviar información de otras personas sin su permiso.

d) Suplantación de identidad. Alguien podría hacerse pasar por el menor utilizando sus perfiles reales, o directamente creando alguno falso para hacerle daño.

e) Sexting. Envío de imágenes, vídeos o textos de carácter sexual.

f) Ciberbullying. Daño intencional, repetido entre iguales que se materializa a través de medios digitales.

g) Grooming. Acercamiento de un adulto a un menor con fines sexuales a través de Internet". 
En algunos casos, estos y otros peligros pueden ser delitos y están regulados como tales (coacción, revelación de secretos, odio, estafas, etc.), pero sobre todo constituyen una fuente objetiva de preocupación entre los internautas. Las recomendaciones habituales en los programas de formación buscan alertar sobre estos riesgos y prevenir de cara a una utilización indebida. Se anima a educar a los menores sobre la privacidad, controlar su tiempo de conexión y utilizar filtros y herramientas para el control parental (Agencia Española de Protección de Datos, 2020). En general, la sobreprotección o el control excesivo se entiende prejudicial, por lo que se recomienda promover una mediación instructiva (Shin y Kang, 2016) o parentalidad positiva (Ramírez-García, Salcines-Talledo y González-Fernández, 2020).

\section{Preocupaciones y necesidades de las familias para educar en las redes sociales}

El horizonte de las preocupaciones familiares se ensancha por efecto de la brecha digital, particularmente la que deriva de la distancia generacional. Efectivamente, los menores se manejan en los entornos digitales con total naturalidad frente a un uso más funcional y limitado entre sus mayores. Según el Pew Research Center (2020) los padres manifiestan que educar es hoy más difícil que en el pasado por causa de las redes sociales. También debe contemplarse el perfil social, e incluso el grado de formación académica o cultural de los internautas, y valorar los niveles y formatos de acceso a las tecnologías. En cualquier caso, no cabe duda de que las familias necesitan ayuda para superar la brecha digital y afrontar adecuadamente las tareas educadoras en la era digital.

Las investigaciones revelan una situación discordante entre el déficit formativo de padres y madres y su deseo de conocer y apropiarse de estos recursos para utilizarlos en favor de una mejor educación de sus hijos. Por un lado, las familias ponen de manifiesto la distancia y la desconfianza que les suscitan los medios digitales, cuyas posibilidades educativas suelen desconocer. Y, al mismo tiempo, muestran gran interés por abarcar este conocimiento y poder aprovecharlo en su favor (Dans, González-Sanmamed y Muñoz, 2019). Por otro lado, cuando se ha encuestado a los hijos, los resultados muestran resultados diversos en cuanto a la permisividad, e incluso se constatan conductas de indiferencia respecto al control parental o bien que este resulta contraproducente (Mullen y Hamilton, 2016). 
Atendiendo a la perspectiva de género, los estudios también son desiguales en sus conclusiones. Así, por ejemplo, existen numerosas prácticas orientadas a favorecer la igualdad relacionadas con el empleo de la web 2.0. en contextos educativos formales (Prendes-Espinosa, GarcíaTudela y Solano-Fernández, 2020). Ahora bien, ¿las familias tratan del mismo modo a las niñas que a los niños en cuanto al empleo de internet y redes sociales?, y ¿ellas y ellos se sienten igual tratados? El macro informe Eu Kids Online no señala diferencias significativas (Smahel et al., 2020), aunque otras investigaciones (Keuhlen, 2019) se refieren a la edad y el género como factores importantes en la forma en que los padres monitorean el uso de Internet de sus hijas e hijos. En estas investigaciones también se indica que quienes tienen buenas experiencias en Internet y una actitud más positiva hacia los adolescentes son más eficaces para controlar el uso de Internet de sus hijos. Los estilos parentales indulgentes se vinculan con actitudes más pacíficas en el ámbito del colegio (Moreno-Ruiz, Estévez, Jiménez y Murgui, 2018). Al contrario, la comunicación ofensiva entre padres e hijos tiene resultados negativos, dado que predice el aumento de la victimización escolar (Garcés, Santoya y Jiménez, 2020).

Tanto el profesorado como los profesionales de la salud insisten en la idea de que es imprescindible favorecer procesos formativos que incluyan a las familias. Así, por ejemplo, la asistencia formativa que deriva de los ámbitos de la salud mental y la pediatría, también incluye la comunicación familiar como una herramienta necesaria para obtener beneficios positivos mediante la navegación compartida, etc. (Sharma, Frederiksen, Malcolm, Rollison y Carter, 2018).

Entre las tendencias de ayuda en la educación digital se encuentra el eparenting o educación parental en línea como "conjunto de prácticas que tienen la finalidad de mejorar las competencias parentales específicas y transversales de familias que requieren un apoyo en el ejercicio y tarea de ser padres y madres, mediante el uso positivo de los recursos tecnológicos y digitales" (Vaquero et al., 2019, p.31). Los enfoques en la orientación familiar pueden ser de carácter educativo o preventivo, de asesoramiento o terapéutico (Ramírez-García, Salcines-Talledo y González-Fernández, 2020). En todos los casos las familias se muestran proclives a recibir formación (2020, p.24), dado que los problemas no se sitúan sólo en el acceso sino también en el alcance y el contenido de las experiencias digitales de sus hijos. 


\section{Método}

\section{Objetivos}

El estudio tenía como principal propósito conocer cuál era el tipo de prohibiciones que padres y madres hacían a sus hijos adolescentes en relación con el uso de Internet, así como identificar si la Red podía suscitar situaciones que derivasen en conflicto familiar. Asimismo, bajo la óptica de los adolescentes usuarios de redes sociales, se buscaba analizar algunos aspectos vinculados a cuestiones parentales en torno a los usos de estas herramientas sociales.

Concretamente, los objetivos establecidos fueron los siguientes:

- Objetivo 1. Conocer cuáles son las principales acciones que los padres y las madres les prohíben hacer a sus hijos cuando están conectados a Internet.

- Objetivo 2. Identificar si en el seno familiar Internet es fuente de discusiones entre los menores y sus padres/madres.

- Objetivo 3. Analizar si el género es una variable que se muestre asociada a la discusión por el uso de internet entre los adolescentes y sus progenitores.

- Objetivo 4. Examinar opiniones de los adolescentes usuarios de redes sociales en torno al papel que debería jugar la familia en aspectos educativos y de control parental.

\section{Participantes}

Sobre una población de 1.792 estudiantes de $4^{\circ}$ curso de enseñanza secundaria de la ciudad de A Coruña, distribuidos en 31 centros educativos (públicos, privados y concertados), se recogieron 1.144 encuestas de 29 instituciones. Para un nivel de confianza del 95\%, y en el supuesto de máxima indeterminación ( $p=q=.50$ y k=2), el margen de error fue de \pm 1.74 .

En relación con el género, el $47.2 \%(n=540)$ eran varones y el $52.8 \%$ $(\mathrm{n}=604)$ mujeres. Según la edad, el alumnado abarcaba un rango de 15 a 18 años, siendo el $41.8 \%(\mathrm{n}=478)$ estudiantes de 15 años, el $45.2 \%$ $(\mathrm{n}=517)$ de 16 años, el 10.6\% $(\mathrm{n}=121)$ de 17 años $\mathrm{y}$, finalmente, el $2.4 \%$ $(\mathrm{n}=28)$ de 18 años.

Según su distribución por tipología de centro, cabe señalar que 416 
de los encuestados eran estudiantes de centros públicos, 689 de centros concertados y 39 de instituciones privadas.

\section{Diseño e instrumento}

Se utilizó un diseño ex post facto basado en el método de encuesta (McMillan y Schumacher, 2005, Cohen y Manion, 2002), empleando un cuestionario estructurado en cinco bloques temáticos: datos de identificación de los participantes; usos de internet y otras tecnologías; utilización de redes sociales; posibilidades de uso de las redes sociales para el aprendizaje escolar y peligros y ventajas de las redes sociales.

Con el fin de asegurar las condiciones psicométricas adecuadas, en la elaboración del cuestionario se atendieron cuestiones como la validez de contenido, de constructo y consistencia interna. El instrumento fue validado por un panel de 5 expertos en metodología de investigación y aplicación de la tecnología a la educación, abordando aspectos como la univocidad, pertinencia e importancia de cada ítem. También se aplicó a un grupo piloto. Asimismo, se efectuaron los correspondientes análisis correlación ítem-total que nos indican si los ítems se relacionan de forma homogénea con la escala a la que pertenecen. Para medir la fiabilidad se utilizó Alfa de Cronbach que arrojó un resultado global de .937.

Para la presente investigación se usaron 7 ítems tomados de los bloques del cuestionario referidos a:

- "Usos de internet y otras tecnologías", donde se planteaba al alumnado dos preguntas con opción de respuesta múltiple:

o Cuando navegas por Internet, según tus padres, ¿qué cosas no puedes hacer? Es decir, señala qué cosas te prohíben tus padres. o ¿Discutes con tus padres por el uso de internet?

- "Opiniones, percepciones y valoraciones sobre las redes sociales", bloque en el que los estudiantes respondieron en base a una escala tipo Likert de cinco puntos: 1 (totalmente en desacuerdo); 2 (en desacuerdo); 3 (sin opinión); 4 (de acuerdo) y 5 (totalmente de acuerdo), en relación con las siguientes cuestiones:

o Los padres deberían recibir formación sobre las redes sociales

o Es necesario que los padres eduquen para un uso correcto en las redes sociales.

o Mi familia se preocupa por los contenidos a los que accedo a través de las redes sociales. 
o Mi familia se preocupa por los contactos que tengo en mis redes sociales.

o Me gustaría compartir algo en una red social con mi familia.

\section{Procedimiento}

Los cuestionarios fueron administrados, en horario escolar, en una única sesión por los investigadores y tras obtener el consentimiento de los directores de los centros educativos y del profesorado. De los 31 centros que conformaban la muestra, tan solo dos de ellos declinaron participar en la investigación. La participación fue voluntaria y se informó a los estudiantes que sus respuestas se mantendrían en el más estricto anonimato. También que toda la información recogida en el estudio se emplearía únicamente con fines académicos.

\section{Análisis de los datos}

Se utilizó estadística descriptiva a través del análisis de frecuencias y porcentajes, así como medidas de tendencia central (media) y dispersión (desviación típica). También se emplearon análisis bivariados no paramétricos mediante tablas de contingencia para variables categóricas con la prueba Chi-Cuadrado. Igualmente se aplicó el test U Mann Whitney para contrastar variables dicotómicas como el género respecto a variables de tipo ordinal.

Para el análisis de los datos se utilizaron los programas SPSS v.20 y para su representación gráfica Microsoft Excel.

\section{Resultados y discusión}

Seguidamente se muestran los resultados derivados de los objetivos establecidos en la investigación e incluidos en líneas precedentes.

En relación con el objetivo 1, centrado en conocer cuáles son las principales acciones que los padres y las madres les prohíben hacer a sus hijos cuando están conectados a Internet, se preguntó a los adolescentes "Cuando navegas por Internet, según tus padres, ¿qué cosas no puedes hacer? es decir, señala qué cosas te prohíben tus padres". Un $76 \%$ de los menores respondieron que sus padres/madres no les prohí- 
ben hacer nada. Este es un dato muy revelador, ya que a partir del mismo se puede inferir que los padres y madres poseen una visión que podría denominarse inocente y poco realista de los riesgos a los que pueden estar expuestos sus hijos si la Red es utilizada de forma poco responsable (Álvarez y Moral, 2020; Díaz-Vicario, Mercader y Gairín, 2019; Cerezo et al., 2016).

Como se aprecia en la figura 1, existen elevados porcentajes de acciones que los padres y madres les permiten hacer a sus hijos cuando están conectados a Internet. No obstante, entre los principales comportamientos vetados nos encontramos con: "comprar algo" (46\%), "dar información personal" (46,9\%), "enviar correos electrónicos a personas desconocidas" (42,6\%).

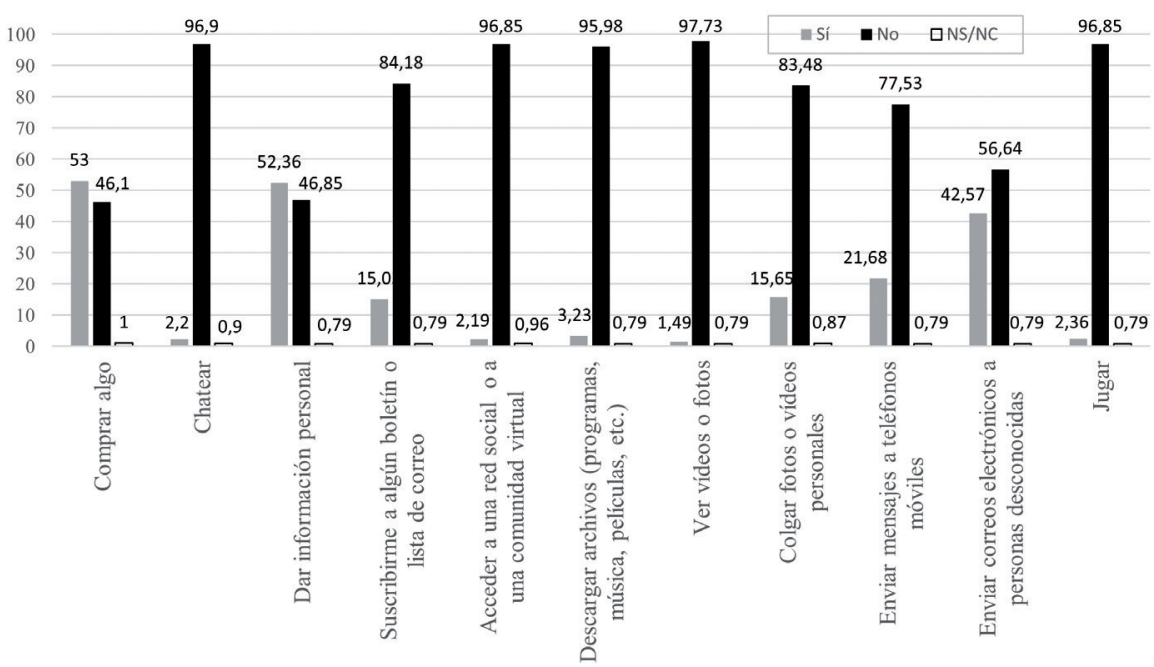

Figura 1. Porcentajes de acciones que los padres y madres permiten y no permiten realizar a sus hijos cuando se conectan a Internet.

Por otra parte, respecto al objetivo 2, relativo a identificar si en el seno familiar Internet es fuente de discusiones entre los menores y sus padres/madres, se ha podido constatar que casi la mitad de los adolescentes encuestados (49\%) han señalado que discuten con sus padres por el uso de internet (figura 2). 


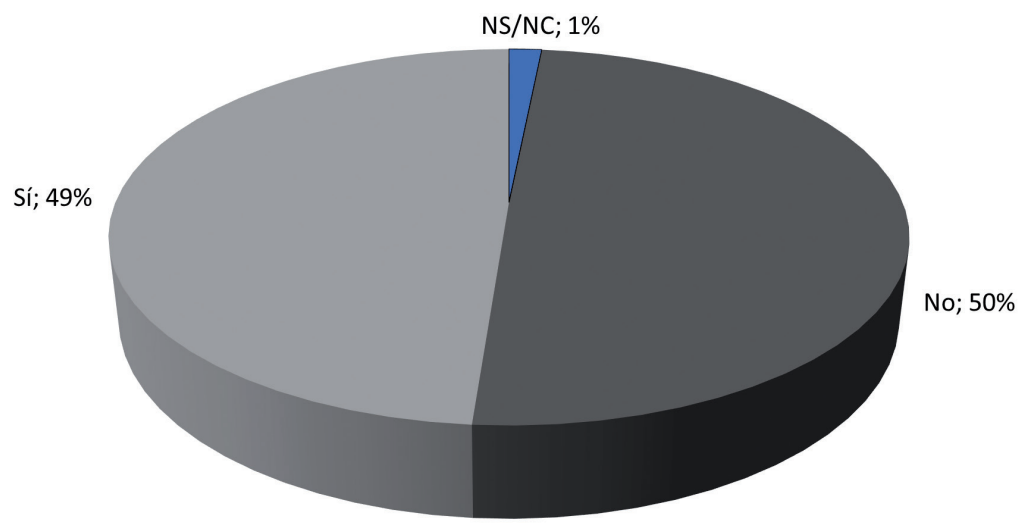

Figura 2. ¿Discutes con tus padres por el uso de Internet?

Si profundizamos en los motivos de discusión por el uso de Internet (tabla 1), se observa para el conjunto de los encuestados, cómo el tiempo conectado es uno de los aspectos que casi siempre (12,2\%) o siempre $(6,6 \%)$ resulta ser motivo de conflicto, seguido del momento del día en el que se produce la conexión (un $5,2 \%$ ha respondido que casi siempre y un $2,1 \%$ que siempre).

Tabla 1

Frecuencias y porcentajes sobre los motivos de discusión entre padres/madres y adolescentes cuando estos últimos se conectan a Internet

\begin{tabular}{|c|c|c|c|c|c|c|}
\hline & $\mathrm{NS} / \mathrm{NC}$ & Nunca & Casi nunca & A veces & Casi siempre & Siempre \\
\hline \multicolumn{7}{|c|}{ Por el tiempo que paso conectado/a } \\
\hline Frecuencia & 566 & 32 & 87 & 245 & 139 & 75 \\
\hline Porcentaje & 49.5 & 2.8 & 7.6 & 21.4 & 12.2 & 6.6 \\
\hline \multicolumn{7}{|c|}{ Por lo que hago mientras estoy conectado/a } \\
\hline Frecuencia & 632 & 309 & 116 & 66 & 13 & 8 \\
\hline Porcentaje & 55.2 & 27.0 & 10.1 & 5.8 & 1.1 & .7 \\
\hline \multicolumn{7}{|c|}{ Por el momento del día en que me conecto } \\
\hline Frecuencia & 623 & 151 & 120 & 166 & 60 & 24 \\
\hline Porcentaje & 54.5 & 13.2 & 10.5 & 14.5 & 5.2 & 2.1 \\
\hline
\end{tabular}

Por otra parte, para comprobar si el género es una variable que se muestre asociada a la discusión por el uso de internet entre los adolescentes y sus progenitores (objetivo 3), es decir, si existe un trato diferente entre los y las jóvenes, se procedió a la realización de una tabla de 
contingencia (ver tabla 2) y, posteriormente, se aplicó el estadístico ChiCuadrado. Los resultados muestran diferencias estadísticamente significativas con un tamaño de efecto elevado [X2(1123, 1)=52.154, $\mathrm{p}<.001$ ]; $V=, 782$. En concreto, las mujeres discuten 7,2 veces más con sus padres que los hombres, lo que hace suponer que los progenitores ejercen un mayor control sobre las chicas frente a los chicos.

Tabla 2

Tabla de contingencia entre las variables género y ¿discutes con tus padres por el uso de Internet?

\begin{tabular}{|c|c|c|c|c|c|}
\hline & & & \multicolumn{2}{|c|}{$\begin{array}{c}\text { ¿Discutes con tus } \\
\text { padres por el uso } \\
\text { de internet? }\end{array}$} & \multirow[t]{2}{*}{ Total } \\
\hline & & & Sí & No & \\
\hline \multirow{8}{*}{ Género } & \multirow{4}{*}{ Alumno } & Recuento & 328 & 202 & 530 \\
\hline & & Frecuencia esperada & 267.6 & 262.4 & 530.0 \\
\hline & & $\begin{array}{l}\% \text { dentro de ¿Discutes con tus } \\
\text { padres por el uso de internet? }\end{array}$ & $57.8 \%$ & $36.3 \%$ & $47.2 \%$ \\
\hline & & Residuos corregidos & 7.2 & -7.2 & \\
\hline & \multirow{4}{*}{ Alumna } & Recuento & 239 & 354 & 593 \\
\hline & & Frecuencia esperada & 299.4 & 293.6 & 593.0 \\
\hline & & $\begin{array}{l}\% \text { dentro de ¿Discutes con tus } \\
\text { padres por el uso de internet? }\end{array}$ & $42.2 \%$ & $63.7 \%$ & $52.8 \%$ \\
\hline & & Residuos corregidos & -7.2 & 7.2 & \\
\hline
\end{tabular}

Por último, para dar respuesta al objetivo 4 relativo a examinar opiniones de los adolescentes usuarios de redes sociales en torno al papel que debería jugar la familia en aspectos educativos y de control parental, se ha elaborado la tabla 3 donde se muestran las frecuencias, porcentajes, media y desviaciones típicas obtenidas. 
Tabla 3

Resultados descriptivos sobre las opiniones de los adolescentes respecto al papel que debería jugar la familia en torno a las redes sociales. Se omiten los valores perdidos.

\begin{tabular}{|c|c|c|c|c|c|c|c|c|c|}
\hline TD* & & & & & & & & & DT \\
\hline$\%$ & $\mathrm{n}$ & $\%$ & $\mathrm{n}$ & $\%$ & $\mathrm{n}$ & $\%$ & $n$ & $\%$ & $\mathrm{NI}$ \\
\hline
\end{tabular}

Los padres deberían recibir formación sobre las $18215.915913 .937432 .727323 .9108 \quad 9.42 .971 .205$ redes sociales Es necesario que los padres eduquen para un 1129.812911 .333629 .434330 .018115 .83 .321 .180 uso correcto de las redes sociales Mi familia se preocupa por los contenidos a los que accedo a tra14712.816614 .531827 .831627 .614913 .03 .141 .225 vés de las redes sociales Mi familia se preocupa por los contactos que 18616.316914 .832328 .227624 .114312 .53 .021 .267 tengo en mis redes sociales Me gustaría

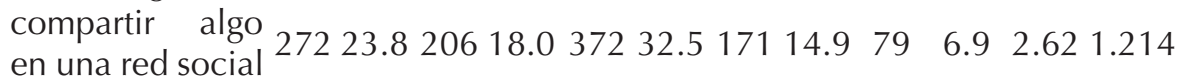
con mi familia

* TD (totalmente en desacuerdo); D (en desacuerdo); SO (sin opinión); A (de acuerdo); TA (totalmente de acuerdo).

Como se puede apreciar en la tabla precedente (tabla 3), las medias más elevadas se han obtenido para los ítems "es necesario que los padres eduquen para un uso correcto de las redes sociales" (media de 3.32) y "mi familia se preocupa por los contactos que tengo en mis redes sociales" (media de 3.14), mientras que la puntuación media más baja se sitúa en el ítem "me gustaría compartir algo en una red social con mi familia" (media de 2.62). Asimismo, tras la aplicación de la prueba $U$ de Mann-Withney, se ha podido identificar que a excepción del ítem "los padres deberían recibir formación sobre las redes sociales", en el resto 
de los mostrados en la tabla 2 se han encontrado diferencias estadísticamente significativas en función del género $(p=.000)$. En concreto, son las mujeres las que han obtenido rangos promedio más elevados.

\section{Conclusiones}

La familia tiene un nuevo conviviente intangible: Internet. Los estudios revelan que, en general, el impacto de la tecnología es positivo, pero resulta imprescindible continuar la indagación acerca de cómo estos dispositivos afectan a la vida familiar (González-Fernández, SalcinesTalledo, Ramírez-García, 2020). Indiscutiblemente, la realidad de un mundo conectado digitalmente exige que los padres y madres organicen la gestión de su propio uso de Internet y, además, que sean capaces de educar la exposición de sus hijos a estas aplicaciones, así como regular su utilización. Esto puede suscitar una serie de beneficios y propicia importantes desafíos. En cualquier caso, las familias viven con inquietud la situación que genera el uso de internet y las redes sociales en la adolescencia, dado que afirman que la educación de los hijos es más difícil hoy que en el pasado y citan la tecnología como uno de los factores desencadenantes (Auxier, Anderson, Perrin y Turner, 2020).

En concreto, los resultados de nuestra investigación se alinean con los expuestos en el macroestudio europeo (Smahel et al., 2020), donde se señala que, en España junto con Suiza, República Checa y Polonia, los padres nunca o casi nunca hablan con sus hijos sobre lo que hacen en Internet. También concuerdan los datos que reflejan diferencias por género, en el sentido de que serían las mujeres quienes recibirían más recomendaciones respecto al uso problemático de internet. Los datos que hemos obtenido van en esta misma línea y, en cierta medida, puede valorarse que esto podría estar en el origen de una mayor conflictividad familiar.

En cualquier caso, las respuestas de los adolescentes ponen de manifiesto que el control por su uso de Internet es de nivel bajo y, si acaso, la causa del enfrentamiento se debe al tiempo de conexión y no al contenido al que acceden. Tal y como se desprende del estudio de SantanaVega, Gómez-Muñoz, Feliciano-García (2019), la falta de regulación parento-filial indica que falla la comunicación familiar. En este sentido, los jóvenes reconocen el papel de la familia como educadora digital, 
quien reclama a su vez esa formación para poder darla a sus hijos. Sin embargo, los adolescentes muestran poca disposición a la hora de compartir sus redes sociales con su familia. Quizás habría que preguntarse $y$, en el futuro analizar, si con este rechazo a compartir redes sociales con su familia se refieren al significado de compartir propio del lenguaje digital (enviar, mostrar, exhibir) y se aleja del verdadero significado de la acepción "participar en algo", donde las conversaciones familiares también incluirían lo que ocurre en Internet.

En suma, cabe recordar que los dispositivos, las conexiones a Internet y las redes sociales también pueden utilizarse como potenciadores de las relaciones personales. La mejora del clima relacional en los hogares con adolescentes guarda relación con una necesaria respuesta coherente al desafío digital. Una posible línea de formación consistiría en potenciar la reflexión de los jóvenes sobre el impacto de sus redes para contribuir a la mejora de la convivencia familiar.

\section{Referencias}

Aaron, L. S. y Lipton, T. (2018). Digital distraction: Shedding light on the 21st-century college classroom. Journal of Educational Technology Systems, 46(3), pp. 363-378. doi: https://doi.org/10.1177/0047239517736876

Álvarez, M. y Moral, M. V. (2020). Phubbing, uso problemático de teléfonos móviles y redes sociales en adolescentes y déficits en autocontrol. Health and addictions, 20(1), 113-125.

Agencia Española de Protección de Datos (2020). Protección del menor en Internet [Versión electrónica en https://www.aepd.es/es/prensa-y-comunicacion/notas-deprensa/la-aepd-publica-recomendaciones-orientadas-evitar-el-acceso]. Madrid: AEPD.

Agencia Española de Protección de Datos y Oficina de Seguridad del Internauta (2016). Protección del menor en Internet [Versión electrónica en https://www.osi.es/es/guiade-privacidad-y-seguridad-en-internet]. Madrid: AEPD.

González, M.; Muñoz, P. y Dans, I. (2017). Factors which motivate the use of social networks by students. Psicothema, 29(2), 204-210. https://doi.org/10.7334/psicothema2016.127.

Dans Álvarez de Sotomayor, I., Muñoz Carril, P. C. y González Sanmamed, M. (2019). Familia y redes sociales: un binomio controvertido. Aula Abierta, 48(2), 183-192. https://doi.org/10.17811/rifie.48.2.2019.183-192.

Dans Álvarez de Sotomayor, I., González-Sanmamed, M. y Muñoz Carril, P. C. (2019). Redes sociales, adolescencia y familia: desafíos y oportunidades. Publicaciones, 49(2), 117-132. https://doi.org/10.30827/publicaciones.v49i2.8527. 
Auxier, B., Anderson, M., Perrin, A. y E. Turner (2020). Parenting Children in the Age of Screens. Pew Research Center. Recuperado de https://www.pewresearch.org/internet/ wp-content/uploads/sites/9/2020/07/PI_2020.07.28_kids-and-screens_FINAL.pdf

Bartsch, M. y Dienlin, T. (2016). Control your Facebook: an analysis of online privacy literacy. Computers in Human Behavior, 56, 147-154. https://doi.org/10.1016/j. chb.2015.11.022

Bernal, A. y König, K. (2017). Percepciones de adolescentes sobre la educación según la identidad personal. Revista Española de Pedagogía, 75 (267), 181-198. doi: https:// doi.org/10.22550/REP75-2-2017-01

Browne, D., May, S., Hurst- Della Pietra, P. et al. (2019). From 'screen time' to the digital level of analysis: protocol for a scoping review of digital media use in children and adolescents. BMJ Open 2019; 9:e032184. doi:10.1136/ bmjopen-2019-032184

Cerezo, F., Arnaiz, P., Giménez, A. M. y Maquilón, J. J. (2016). Conductas de ciberadicción y experiencias de cyberbullying entre adolescentes. Anales de Psicología, 32(3), 761-769. http://dx.doi.org/10.6018/analesps.32.3.217461

Christofides, E., Muise, A. y Desmarais, S. (2012). Risky Disclosures on Facebook. The Effect of Having a Bad Experience on Online Behavior. Journal of Adolescent Research, 27(6), pp. 714-731.

Cohen, L. y Manion, L. (2002). Métodos de investigación educativa. Madrid: La Muralla.

Díaz-Vicario, A., Mercader, C., y Gairín, J. (2019). Uso problemático de las TIC en adolescentes. Revista Electrónica de Investigación Educativa, 21(7), 1-11. https.//doi. org/10.24320/redie.2019.21.e07.1882

Gallardo, I. M., De Castro, A. y Saiz, H. (2020). Interacción y uso de tecnologías en los procesos de enseñanza y aprendizaje. Educatio siglo XXI: Revista de la Facultad de Educación, 38 (1), pp. 119-137

Garcés, M., Santoya, Y. y Jiménez, J. (2020). Influencia de la comunicación familiar y pedagógica en la violencia escolar. Comunicar, 63, 77-86. https://doi.org/10.3916/ C63-2020-07

Garrido, M., Busquet, J. y Munté, R. A. (2016). De las TIC a las TRIC. Estudio sobre el uso de las TIC y la brecha digital entre adultos y adolescentes en España. Anàlisi. Quaderns de Comunicació i Cultura, (54), 44-57. doi: http://dx.doi.org/10.7238/a. v0i54.2953

Harper, B. (2018). Technology and teacher-student interactions: A review of empirical research. Journal of Research on Technology in Education, 50(3), pp. 214-225. doi: https://doi.org/10.1080/15391523.2018.1450690

Hobbs, R. (2010). Digital and Media Literacy: A Plan of Action. A White Paper on the Digital and Media Literacy Recommendations of the Knight Commission on the Information Needs of Communities in a Democracy. [Versión electrónica en https://eric. ed.gov/?id=ED523244]. Washington, DC.: Aspen Institute.

Keuhlen, K. D., Donald, K., Falbo, R., Lekuti, Y., Marroquin, L., \& Ladd, L. (2019). Stop! Collaborate and Listen: A Content Analysis of Peer-Reviewed Articles Investigating Parenting Strategies for Managing Adolescent Internet Use. Contemporary Family Therapy, pp. 1-12. doi: https://doi.org/10.1007/s10591-019-09510-z 
Internet y redes sociales: un desafío a la convivencia familiar

isabel Dans Álvarez De Sotomayor y Pablo César Muñoz Carril

Lozano-Blasco, R. y Cortés-Pascual, A. (2020). Problematic Internet uses and depression in adolescents: A meta-analysis. [Usos problemáticos de Internet y depresión en adolescentes: Meta-análisis]. Comunicar, 63, pp. 109-120. doi: https://doi.org/10.3916/ C63-2020-10

Manca, S. y Ranieri, M. (2015). Implications of social network sites for teaching and learning. Where we are and where we want to go, Educ. Inf. Technol. 22 (2), pp. 605-622, doi: https://doi.org/10.1007/ s10639-015-9429-x

Mauri, M., Cipresso, P., Balgera, A., Villamira, M. y Riva, G. (2011). Why is Facebook so successful? Psychophysiological measures describe a core flow state while using Facebook. Cyberpsychol Behavior Social Network, 14(12), pp. 723-31. doi: 10.1089/ cyber.2010.0377.

McMillan, J. y Schumacher, S. (2005). Investigación educativa. Madrid: Pearson Addison Wesley.

Michavila, Abad y García (2019). Impacto de las pantallas en la vida familiar. Madrid: Empantallados.

Min, J. (2016). Personal information concerns and provision in social network sites: Interplay between secure preservation and true presentation. Journal of the Association for Information Science and Technology, 67(1), 26-42. doi: http://dx.doi.org/10.1002/asi.23376

Moreno-Ruiz, D., Estévez, E., Jiménez, T., Murgui, S. (2018). Parenting Style and Reactive and Proactive Adolescent Violence: Evidence from Spain. Int I Environ Res Public Health. 15(12):2634. doi:10.3390/ijerph15122634

Mullen, C. y Hamilton, N. F. (2016). Adolescents' response to parental Facebook friend requests: The comparative influence of privacy management, parent-child relational quality, attitude and peer influence. Computers in Human Behavior, 60, pp. 165-172. doi: http://dx.doi.org/10.1016/j.chb.2016.02.026.

Pew Research Center (2018). How Teens and Parents Navigate Screen Time and Device Distractions. Recuperado de http://www.pewinternet.org/2018/08/22/how-teensand-parents-navigate-screen-time-and-device-distractions/

Prendes-Espinosa, M., García-Tudela, P. y Sodano-Fernández, I. (2020). Igualdad de género y TIC en contextos educativos formales: Una revisión sistemática. Comunicar: Revista científica iberoamericana de comunicación y educación, (63), pp. 9-20. doi: https://doi.org/10.3916/C63-2020-01,

Ramírez-García, A., Salcines-Talledo, I. y González-Fernández, N. (2020). Los dispositivos móviles en el hogar. Interés formativo de las familias españolas. REOP-Revista Española de Orientación y Psicopedagogía, 31(1), pp. 43-61. doi: https://doi. org/10.5944/reop.vol.31.num.1.2020.27286

Santana-Vega, L. E., Gómez-Muñoz, A. M. y Feliciano-García, L. (2019). Uso problemático del móvil, fobia a sentirse excluido y comunicación familiar de los adolescentes. Comunicar: Revista Científica de Comunicación y Educación, 27(59), 39-47. doi:https://doi.org/10.3916/C59-2019-04

Selwyn, N. (2016). Is technology good for education? Cambridge, UK: Polity Press.

Serrano-Puche, J. (2016). Internet y emociones: nuevas tendencias en un campo de investigación emergente. Comunicar, 46, pp. 19-26. doi: http://dx.doi.org/10.3916/ C46-2016-02 
Sharma, A., Frederiksen, B., Malcolm, N., Rollison, J. y Carter, M. (2018). Community education and engagement in family planning: updated systematic review. American journal of preventive medicine, 55(5), pp. 747-758. doi: https://doi.org/10.1016/j. amepre.2018.06.022

Shin, W. y Kang, H. (2016). Adolescents' privacy concerns and information disclosure online: The role of parents and the Internet. Computers in Human Behavior. 54, pp. 114-123. http://doi. org/10.1016/j.chb.2015.07.062.

Smahel, D., Machackova, H., Mascheroni, G., Dedkova, L., Staksrud, E., Ólafsson, K., Livingstone, S. y Hasebrink, U. (2020). EU Kids Online 2020: Survey results from 19 countries. EU Kids Online. doi: https://doi.org/10.21953//se.47fdeqj01 ofo.

Terán, A. (2019). Ciberadicciones. Adicción a las nuevas tecnologías (NTIC). En: AEPap (ed.). Congreso de Actualización Pediatría 2019. Madrid: Lúa Ediciones 3.0, pp. 131 141.

UNESCO (2011). Alfabetización mediática e informacional: currículum para profesores [Versión electrónica en http://unesco.mil-for-teachers.unaoc.org/glosario-determinos/?lang=es]

Vanderhoven, E., Schellens, T. y Valcke, M. (2014). Educating Teens about the Risks on Social Network Sites. An intervention study in Secondary Education. Comunicar, 43, pp. 123-132.

Vaquero, E., Suárez, A., Fernández-Rodrigo, L., Rodrigo, M. J., y Balsells Bailón, M. Àngels. (2019). E-parenting: una revisión sistemática de la literatura. Edutec. Revista Electrónica De Tecnología Educativa, (68), pp. 30-41. doi: https://doi.org/10.21556/ edutec.2019.68.1313

\section{Agradecimientos}

Este trabajo se ha elaborado en el marco del proyecto de investigación titulado: "Ecologías de aprendizaje en la era digital: nuevas oportunidades para la formación del profesorado de educación secundaria" (ECO4 LEARN-SE), parcialmente financiado por el Ministerio de Ciencia, Innovación y Universidades (Referencia RTI2018-095690-B-I00). 
